\title{
COMPUTING A LOGARITHM OF A UNITARY MATRIX WITH GENERAL SPECTRUM
}

\author{
TERRY A. LORING
}

\begin{abstract}
We analyze an algorithm for computing a skew-Hermitian logarithm of a unitary matrix. This algorithm is very easy to implement using standard software and it works well even for unitary matrices with no spectral conditions assumed. Certain examples, with many eigenvalues near -1 , lead to very non-Hermitian output for other basic methods of calculating matrix logarithms. Altering the output of these algorithms to force an Hermitian output creates accuracy issues which are avoided in the considered algorithm.

A modification is introduced to deal properly with the $J$-skew symmetric unitary matrices. Applications to numerical studies of topological insulators in two symmetry classes are discussed.
\end{abstract}

\section{INTRODUCTION}

While all invertible matrices have a logarithm, indeed many logarithms, the unitary matrices have the nicest logarithms. Every unitary matrix $U$ has a skew-Hermitian logarithm. Indeed, when $U$ is unitary, the conditions

$$
-\pi<K \leq \pi, \quad e^{i K}=U
$$

specify $K$ uniquely. Still working abstractly, we may take advantage of the finiteness of the spectrum of $U$ when discussing additional symmetries. For each $U$ there is a complex polynomial so that $K=p(U)$ which means, for example, that when $U$ is $J$ skew-symmetric then so must be $K$.

We may regard $U^{*}=U^{-1}$ as a symmetry, but the non-linearity of the unit circle inevitably makes this symmetry a little different from that of being Hermitian $\left(A=A^{*}\right)$ or symmetric $\left(A^{\top}=A\right)$. In the case of a matrix being Hermitian, if numerical errors start to creep in, we simple replace $A$ by $\frac{1}{2} A+\frac{1}{2} A^{*}$ in $\mathcal{O}\left(n^{2}\right)$ time and have again $A^{*}=A$ exactly. The unitary part of the polar decomposition can be used when $U^{*} U$ is only close to $I$ but it takes more that $\mathcal{O}\left(n^{2}\right)$ time to compute and the result is not exactly unitary. For this reason we need to consider errors in $\left\|U^{*} U-I\right\|$ a bit larger than machine precision.

Remark. We are using $U^{*}$ to denote conjugate-transpose of a matrix. This would be denoted with a dagger in physics. For just the conjugate we use $\bar{U}$.

2000 Mathematics Subject Classification. 47B15,65F60.

Key words and phrases. MATLAB, LAPACK, matrix logarithm, matrix exponential, Schur decomposition, functional calculus, normal matrices, unitary matrices, self-dual matrices, Floquet Hamiltonian. 
What is called self-dual [9] in physics is called J-skew-symmetric [3] or skew Hamiltonian [2] in computer science.

Finally, what applied mathematicians call matrix functions are called, in pure math, applications of the functional calculus.

The algorithm discussed here arose in a numerical study in condensed matter physics [10]. In that situation $\left\|U^{*} U-I\right\| \approx \frac{1}{2}$ was typical. Such a matrix $U$ is still very well conditioned, so one would expect almost any algorithm for computing a logarithm to perform well, at least most of the time. However the study in question had a timereversal symmetry that, through a variation on Kramers pairs, caused the approximate unitaries in question to have multiplicity at least two in every eigenvalue. Otherwise the approximate unitaries were free to have arbitrary spectrum within the unit circle. Some reflection makes one realize that whatever branch of logarithm one uses, a degenerate eigenvalue on the branch point has the potential to cause trouble. This is true whether or not the degeneracy in the spectrum is caused by a symmetry or by very bad luck.

We are not claiming it is hard to find $K$ with $e^{i K} \approx U$. We are interested in achieving $e^{i K} \approx U$ and $K^{*}=K$ at the same time. Moreover we want an algorithm that can be easily analyzed and also easily modified to take into account additional symmetries.

To be consistent, we consider the branch of logarithm that has the imaginary part of $\log (\lambda)$ in the interval $(-\pi, \pi]$, so in particular $\log (-1)=i \pi$. We are mainly concerned with the operator norm

$$
\|A\|=\sup _{\mathbf{v} \neq 0} \frac{\|A \mathbf{v}\|_{2}}{\|\mathbf{v}\|_{2}}
$$

We also will use the Frobenius norm, i.e. the un-normalized Hilbert-Schmidt norm

$$
\|A\|_{\mathrm{F}}=\sqrt{\sum_{j, k=1}^{n}\left|A_{j k}\right|^{2}} .
$$

Recall the bounds $\|A\| \leq\|A\|_{\mathrm{F}} \leq \sqrt{n}\|A\|$.

One easy solution, that often works well for logarithms of well-conditioned matrices, starts by diagonalizing $U$ via an invertible.

\section{Algorithm 1.}

(1) Compute a diagonalization, $W$ invertible and $T$ diagonal with $W T W^{-1} \approx U$.

(2) Create a diagonal unitary matrix $D$ via $D_{j j}=T_{j j} /\left|T_{j j}\right|$.

(3) Compute $H_{0}=W \log (D) W^{-1}$.

(4) Output: $H=\frac{1}{2} H_{0}^{*}+\frac{1}{2} H_{0}$.

This fails badly in special cases, even for small matrices. Given

$$
U=\left[\begin{array}{cc}
-1 & 0 \\
0 & -1
\end{array}\right]
$$


we might consider the approximate diagonalization

$$
W=\left[\begin{array}{ll}
1 & \alpha \\
0 & 1
\end{array}\right], \quad D=\left[\begin{array}{cc}
-1 & 0 \\
0 & \exp (-\pi i+\theta i)
\end{array}\right]
$$

to be rather good if $\theta>0$ is small, even if $\alpha>0$ is rather large. Indeed

$$
\begin{aligned}
W D W^{-1}-U & =\left[\begin{array}{cc}
-1 & \alpha(1+\exp (-\pi i+\theta i)) \\
0 & \exp (-\pi i+\theta i)
\end{array}\right]-U \\
& =(1+\exp (-\pi i+\theta i))\left[\begin{array}{cc}
0 & \alpha \\
0 & 1
\end{array}\right]
\end{aligned}
$$

which has norm

$$
|1+\exp (-\pi i+\theta i)| \sqrt{1+\alpha^{2}}
$$

However, if we set

$$
\begin{aligned}
K_{0} & =-i W \log (D) W^{-1} \\
& =\left[\begin{array}{ll}
1 & \alpha \\
0 & 1
\end{array}\right]\left[\begin{array}{cc}
\pi & 0 \\
0 & -\pi+\theta
\end{array}\right]\left[\begin{array}{cc}
1 & -\alpha \\
0 & 1
\end{array}\right] \\
& =\left[\begin{array}{cc}
\pi & -\pi \alpha+\theta \alpha \\
0 & -\pi+\theta
\end{array}\right]\left[\begin{array}{cc}
1 & -\alpha \\
0 & 1
\end{array}\right] \\
& =\left[\begin{array}{cc}
\pi & -2 \pi \alpha+\theta \alpha \\
0 & -\pi+\theta
\end{array}\right]
\end{aligned}
$$

we note that this is not close to the actual logarithm, which is diagonal. It is not close to any branch of logarithm applied to $U$. When we enforce the symmetry by $K=\frac{1}{2} K_{0}^{*}+\frac{1}{2} K_{0}$, we obtain

When $\alpha=1$ we have

$$
K=\left[\begin{array}{cc}
\pi & -\pi \alpha+\frac{1}{2} \theta \alpha \\
-\pi \alpha+\frac{1}{2} \theta \alpha & -\pi+\theta
\end{array}\right]
$$

and yet

$$
\lim _{\theta \rightarrow 0}\left\|W D W^{-1}-U\right\|=0
$$

where

$$
\left[\begin{array}{cc}
\pi & -\pi \alpha+\frac{1}{2} \theta \\
-\pi \alpha+\frac{1}{2} \theta & -\pi+\theta
\end{array}\right] \rightarrow K_{1}
$$

$$
K_{1}=\left[\begin{array}{cc}
\pi & -\pi \\
-\pi & -\pi
\end{array}\right]
$$

Working numerically, we find

$$
\lim _{\theta \rightarrow 0}\left\|e^{i K}-U\right\|=\left\|e^{i K_{1}}-U\right\| \approx 1.2114
$$

which is dramatically off.

Examining the methods for computing matrix exponentials, both good and bad, in [18], we notice methods based on the Schur decomposition. In theory, the Schur decomposition of a normal matrix will result in a an upper triangular factor that is actually diagonal. 
So almost normal should lead to an almost diagonal factor, but this is too naive an approach. However we are not concerned with general almost normal matrices just now, only the nice special case of almost unitary matrices. A naive approach using the Schur decomposition will work well in this specialized situation.

The logm function in MATLAB is optimized to so that $H=\operatorname{logm}(U)$ leads to $\operatorname{expm}(H)$ being very close to $U$, even when $U$ is badly conditioned. It is not optimized to convert the approximate relation $U^{*} U \approx I$ into the approximate relation $H^{*} \approx-H$. The SchurParlett algorithm [6] behind logm and funm is primarily intended for entire functions. It can behaive unexpectedly when eigenvalues are at and near branch points.

Algorithm 2.

(1) Compute logarithm $H_{0}$ of $U$ via logm.

(2) Output: $H=\frac{1}{2} H_{0}^{*}+\frac{1}{2} H_{0}$.

A far more ambitious project would be to find algorithms for matrix functions that, given almost normal matrices as input, lead to almost normal matrices on output. The subject of almost normal matrices is discussed in a recent survey [5] and is full of subtle problems.

\section{Schur FACTORIZATION OF NEAR UNitARY MATRICES}

We define the deviation from unitary to be the number $\left\|U^{*} U-I\right\|$.

In finite precision arithmetic, we can expect the deviation from unitary to almost never equal zero. Not surprisingly this error is easy to handle.

Lemma 1. Suppose $U$ is in $\mathbf{M}_{n}(\mathbb{C})$ and $U \mathbf{v}=\lambda \mathbf{v}$ for some unit vector $\mathbf{v}$ and scalar $\lambda$. Then

$$
\left.|| \lambda\right|^{2}-1 \mid \leq\left\|U^{*} U-I\right\|
$$

Proof. Let $P$ denote the orthogonal projection on the one-dimensional space spanned by $\lambda$. Then

$$
\begin{aligned}
\left.|| \lambda\right|^{2}-1 \mid & =\left\|\left(|\lambda|^{2}-1\right) P\right\| \\
& =\left\|P\left(U^{*} U-I\right) P\right\| \\
& \leq\left\|U^{*} U-I\right\| .
\end{aligned}
$$

Remark 2. Notice then

$$
\sqrt{1-\left\|U^{*} U-I\right\|} \leq|\lambda| \leq \sqrt{1+\left\|U^{*} U-I\right\|}
$$

so that as long as $\left\|U^{*} U-I\right\|<1$ we have

$$
|| \lambda|-1| \leq\left\|U^{*} U-I\right\| .
$$

Lemma 3. Suppose $T$ in $\mathbf{M}_{n}(\mathbb{C})$ is upper triangular and let $D$ denote the diagonal matrix corresponding to the diagonal of $T$. Then

$$
\|T-D\|_{\mathrm{F}} \leq \sqrt{2(n-1)}\left\|T^{*} T-I\right\|^{\frac{1}{2}}
$$


Proof. We know that the $T_{j j}$ are all eigenvalues of $T$ so

$$
\left.|| T_{j j}\right|^{2}-1 \mid \leq\left\|T^{*} T-I\right\| \text {. }
$$

Every element on the diagonal of a matrix has absolute value at most the norm of that matrix. Applied to $T^{*} T-I$ this tells us

$$
\left\|T^{*} T-I\right\| \geq\left.\left.\left|-1+\sum_{k=1}^{j}\right| T_{k j}\right|^{2}\left|\geq \sum_{k=1}^{j-1}\right| T_{k j}\right|^{2}-\left.|| T_{j j}\right|^{2}-1 \mid
$$

so

$$
\sum_{k=1}^{j-1}\left|T_{k j}\right|^{2} \leq 2\left\|T^{*} T-I\right\|
$$

Summing these error bounds we learn

$$
\left(\|T-D\|_{\mathrm{F}}\right)^{2} \leq 2(n-1)\left\|T^{*} T-I\right\| .
$$

Remark 4 . We get here the estimate

$$
\|T-D\|_{\mathrm{F}} \leq \sqrt{2(n-1)}\left\|T^{*} T-I\right\|_{\mathrm{F}}^{\frac{1}{2}}
$$

which we can compare to Henrici's estimate [1]

$$
\|T-D\|_{\mathrm{F}} \leq\left(\frac{n^{3}-n}{12}\right)^{\frac{1}{2}}\left\|T^{*} T-T T^{*}\right\|_{\mathrm{F}}^{\frac{1}{2}} .
$$

As expected, we are getting a better estimate for almost unitary matrices than works in the more general case of almost normal matrices.

Lemma 5. Suppose $T$ in $\mathbf{M}_{n}(\mathbb{C})$ is upper triangular with $\left\|T^{*} T-I\right\|<1$. If we let $D$ denote the diagonal matrix with

$$
D_{j j}=\frac{1}{\left|T_{j j}\right|} T_{j j}
$$

then

$$
\|T-D\| \leq(\sqrt{2(n-1)}+1)\left\|T^{*} T-I\right\|^{\frac{1}{2}} .
$$

Proof. The previous lemma shows that with $E$ diagonal and $E_{j j}=T_{j j}$ we have

$$
\|T-E\| \leq\|T-E\|_{\mathrm{F}} \leq \sqrt{2(n-1)}\left\|T^{*} T-I\right\|^{\frac{1}{2}} .
$$

Dealing with diagonal matrices is easy, and we find

$$
\|D-E\|=\sup _{j}\left|T_{j j}-\frac{1}{\left|T_{j j}\right|} T_{j j}\right| \leq\left\|T^{*} T-I\right\|
$$


by Remark 4 so

$$
\begin{aligned}
\|T-D\| & \leq \sqrt{2(n-1)}\left\|T^{*} T-I\right\|^{\frac{1}{2}}+\left\|T^{*} T-I\right\| \\
& \leq(\sqrt{2(n-1)}+1)\left\|T^{*} T-I\right\|^{\frac{1}{2}} .
\end{aligned}
$$

Theorem 6. Suppose $U$ is in $\mathbf{M}_{n}(\mathbb{C})$ and that unitary $Q$ and upper-triangular matrix $T$ are a Schur factorization for $U$, meaning $U=Q T Q^{*}$. If we define $D$ to be the diagonal unitary matrix with

then

$$
D_{j j}=\frac{1}{\left|T_{j j}\right|} T_{j j}
$$

$$
\left\|U-Q D Q^{*}\right\| \leq(\sqrt{2(n-1)}+1)\left\|U^{*} U-I\right\|^{\frac{1}{2}} .
$$

Proof. We have

and

$$
\left\|T^{*} T-I\right\|=\left\|Q^{*}\left(U^{*} U-I\right) Q\right\|=\left\|U^{*} U-I\right\|
$$

$$
\|T-D\|=\left\|Q^{*}\left(U-Q D Q^{*}\right) Q\right\|=\left\|U-Q D Q^{*}\right\|
$$

so this follows immediately.

\section{Diagonalizing matrices that are Close to unitary}

We may face a matrix $U$ where $U^{*} U$ is as close to $I$ as can be expected within the constraints of machine precision. For Hermitian matrices, the eigensolvers in LAPACK produce eigenvectors that are "always nearly orthogonal to working precision" [1, §4.7.1]. No comparable promise is made in other eigensolvers, but off-the-shelf algorithm such as ZGEES in LAPACK computing a Schur factorization form a good substitute. We get a simple algorithm for finding a unitary eigensolver for a unitary matrix.

\section{Algorithm 3.}

(1) Compute a Schur factorization, $Q$ unitary and $T$ upper-triangular, $Q T Q^{*} \approx U$.

(2) Create a unitary diagonal matrix $D$ via $D_{j j}=T_{j j} /\left|T_{j j}\right|$.

(3) Compute $Q \log (D) Q^{*}$.

If $\left\|U^{*} U-I\right\|$ is a larger than machine precision, then we can compute the unitary part of $U$ and proceed as above. For simplicity of programming, we will compute the polar decomposition via the singular value decomposition. We are not advocating this method, heeding the warnings in [12].

Algorithm 4.

(1) Set $V=U\left(U^{*} U\right)^{-\frac{1}{2}}$.

(2) Compute a Schur factorization, $Q$ unitary and $T$ upper-triangular, $Q T Q^{*} \approx V$.

(3) Create a unitary diagonal matrix $D$ via $D_{j j}=T_{j j} /\left|T_{j j}\right|$.

(4) Compute $Q \log (D) C^{*}$; 
COMPUTING A LOGARITHM OF A UNITARY MATRIX WITH GENERAL SPECTRUM

\begin{tabular}{|c|c|c|c|c|c|c|}
\hline & $\begin{array}{c}\text { deviation } \\
\text { from unitary }\end{array}$ & \multicolumn{5}{|c|}{ Backwards Error $\left\|e^{-i H}-U\right\|$} \\
\hline$n$ & $\left\|U^{*} U-I\right\|$ & Algorithm 1 & Algorithm 2 & Algorithm 3 & Algorithm 4 & Algorithm 5 \\
\hline \hline 8 & $4.11082 \mathrm{e}-15$ & 0.12285 & 0.12340 & $4.30902 \mathrm{e}-15$ & $4.41189 \mathrm{e}-15$ & $4.13976 \mathrm{e}-15$ \\
\hline 16 & $5.02961 \mathrm{e}-15$ & 0.04407 & 0.04465 & $6.31606 \mathrm{e}-15$ & $6.47906 \mathrm{e}-15$ & $6.13171 \mathrm{e}-15$ \\
\hline 32 & $6.33082 \mathrm{e}-15$ & 0.09286 & 0.09099 & $9.36391 \mathrm{e}-15$ & $9.30067 \mathrm{e}-15$ & $8.99073 \mathrm{e}-15$ \\
\hline 64 & $1.10432 \mathrm{e}-14$ & 0.01952 & 0.01641 & $1.38378 \mathrm{e}-14$ & $1.39124 \mathrm{e}-14$ & $1.32675 \mathrm{e}-14$ \\
\hline 128 & $1.34734 \mathrm{e}-14$ & 0.01999 & 0.02239 & $2.29980 \mathrm{e}-14$ & $2.32533 \mathrm{e}-14$ & $2.26790 \mathrm{e}-14$ \\
\hline 256 & $3.19324 \mathrm{e}-14$ & 0.06131 & 0.06158 & $4.49885 \mathrm{e}-14$ & $4.31729 \mathrm{e}-14$ & $4.42639 \mathrm{e}-14$ \\
\hline
\end{tabular}

TABLE 1. We compare algorithms that produce matrices $H$ with $H^{*}=H$ exactly but with $e^{i H} \approx U$ with varying accuracy. These are run on unitary matrices with at least two eigenvalues near -1 . For each $n$ the data shown are averaged over 30 test matrices. The noise variable in the code in the Appendix was set to $10^{-15} n^{-0.56}$. The exponent -0.56 was determined to keep the deviation from unitary close to constant across different matrix sizes.

Our focus is on $U$ with deviation from unitary in the range $(0,0.1)$. Newton's method of approximating the unitary part of $U$ is very effective in this situation. Newton's method here sets $V=U$ and iterates the replacement $V \leftarrow \frac{1}{2}\left(V+\left(V^{-1}\right)^{*}\right)$. For our purposes, it makes sense to use two interations.

Algorithm 5.

(1) Set $V_{1}=\frac{1}{2}\left(U+\left(U^{-1}\right)^{*}\right)$.

(2) Set $V=\frac{1}{2}\left(V_{1}+\left(V_{1}^{-1}\right)^{*}\right)$.

(3) Compute a Schur factorization, $Q$ unitary and $T$ upper-triangular, $Q T Q^{*} \approx V$.

(4) Create a unitary diagonal matrix $D$ via $D_{j j}=T_{j j} /\left|T_{j j}\right|$.

(5) Compute $Q \log (D) Q^{*}$.

One could compute when to stop the iterations for best accuracy, following, [12]. As simpler methods work here, where we have such well-conditioned matrices, we do not pursue this option.

Even one iteration of Newton's method has advantages.

Lemma 7. Suppose $U$ is in $\mathbf{M}_{n}(\mathbb{C})$ and $\left\|U^{*} U-I\right\| \leq \frac{3}{4}$. If

$$
V=\frac{1}{2}\left(U+\left(U^{-1}\right)^{*}\right)
$$

then

$$
\left\|V^{*} V-I\right\| \leq\left\|U^{*} U-I\right\|^{2}
$$

and

$$
\|U-V\| \leq\left\|U^{*} U-I\right\|
$$




\begin{tabular}{|c|c|c|c|c|c|c|}
\hline & $\begin{array}{c}\text { deviation } \\
\text { from unitary }\end{array}$ & \multicolumn{5}{|c|}{ Time } \\
\hline$n$ & $\left\|U^{*} U-I\right\|$ & Algorithm 1 & Algorithm 2 & Algorithm 3 & Algorithm 4 & Algorithm 5 \\
\hline \hline 8 & $4.11082 \mathrm{e}-15$ & $0.00013 \mathrm{~s}$ & $0.00190 \mathrm{~s}$ & $0.00009 \mathrm{~s}$ & $0.00014 \mathrm{~s}$ & $0.00016 \mathrm{~s}$ \\
\hline 16 & $5.02961 \mathrm{e}-15$ & $0.00034 \mathrm{~s}$ & $0.00618 \mathrm{~s}$ & $0.00024 \mathrm{~s}$ & $0.00036 \mathrm{~s}$ & $0.00037 \mathrm{~s}$ \\
\hline 32 & $6.33082 \mathrm{e}-15$ & $0.00139 \mathrm{~s}$ & $0.02104 \mathrm{~s}$ & $0.00104 \mathrm{~s}$ & $0.00146 \mathrm{~s}$ & $0.00145 \mathrm{~s}$ \\
\hline 64 & $1.10432 \mathrm{e}-14$ & $0.00877 \mathrm{~s}$ & $0.05853 \mathrm{~s}$ & $0.00719 \mathrm{~s}$ & $0.00926 \mathrm{~s}$ & $0.00886 \mathrm{~s}$ \\
\hline 128 & $1.34734 \mathrm{e}-14$ & $0.06111 \mathrm{~s}$ & $0.13750 \mathrm{~s}$ & $0.05442 \mathrm{~s}$ & $0.06843 \mathrm{~s}$ & $0.06218 \mathrm{~s}$ \\
\hline 256 & $3.19324 \mathrm{e}-14$ & $0.33397 \mathrm{~s}$ & $0.73867 \mathrm{~s}$ & $0.29425 \mathrm{~s}$ & $0.35710 \mathrm{~s}$ & $0.32437 \mathrm{~s}$ \\
\hline
\end{tabular}

TABLE 2. Timing data for the algorithms and data set from Table 1, Times were computed in a non-parallel environment.

Proof. Let $U=D \Omega$ be the alternate-side polar decomposition of $U$, so $\Omega$ is unitary and $D$ is positive semi-definite. Then

$$
\left\|U^{*} U-I\right\|=\left\|\Omega^{*} D^{2} \Omega-I\right\|=\left\|D^{2}-I\right\|=\sup _{\lambda \in \sigma(D)}\left|\lambda^{2}-1\right|
$$

and

$$
\left\|V^{*} V-I\right\|=\left\|\left|\frac{1}{2}\left(D+D^{-1}\right)\right|^{2}-I\right\|=\sup _{\lambda \in \sigma(D)}\left|\frac{1}{4}\left(\lambda+\lambda^{-1}\right)^{2}-1\right|
$$

and

$$
\|U-V\|=\left\|D-\frac{1}{2}\left(D+D^{-1}\right)\right\|=\sup _{\lambda \in \sigma(D)}\left|\lambda-\frac{1}{2}\left(\lambda+\lambda^{-1}\right)\right| .
$$

This lemma reduces to routine algebra, showing that

$$
\frac{1}{2} \leq \lambda \leq \frac{\sqrt{7}}{2}
$$

implies

$$
\left|\frac{1}{4}\left(\lambda+\lambda^{-1}\right)^{2}-1\right| \leq\left|\lambda^{2}-1\right|^{2}
$$

and

$$
\left|\lambda-\frac{1}{2}\left(\lambda+\lambda^{-1}\right)\right| \leq\left|\lambda^{2}-1\right|
$$

Theorem 8. Suppose $U$ is in $\mathbf{M}_{n}(\mathbb{C})$ with $\left\|U^{*} U-I\right\| \leq \frac{3}{4}$ and let

$$
V=\frac{1}{2}\left(U+\left(U^{-1}\right)^{*}\right) \text {. }
$$




\begin{tabular}{|c|c|c|c|c|c|c|}
\hline & $\begin{array}{c}\text { deviation } \\
\text { from unitary }\end{array}$ & \multicolumn{5}{|c|}{ Backwards Error $\left\|e^{-i H}-U\right\|$} \\
\hline$n$ & $\left\|U^{*} U-I\right\|$ & Algorithm 1 & Algorithm 2 & Algorithm 3 & Algorithm 4 & Algorithm 5 \\
\hline \hline 8 & $1.19608 \mathrm{e}-05$ & 0.33294 & 0.33294 & $9.42901 \mathrm{e}-06$ & $5.98042 \mathrm{e}-06$ & $5.98042 \mathrm{e}-06$ \\
\hline 16 & $1.27157 \mathrm{e}-05$ & 0.18364 & 0.18364 & $1.01912 \mathrm{e}-05$ & $6.35784 \mathrm{e}-06$ & $6.35784 \mathrm{e}-06$ \\
\hline 32 & $1.25555 \mathrm{e}-05$ & 0.24113 & 0.24113 & $1.01824 \mathrm{e}-05$ & $6.27775 \mathrm{e}-06$ & $6.27775 \mathrm{e}-06$ \\
\hline 64 & $1.21902 \mathrm{e}-05$ & 0.21304 & 0.21304 & $9.96141 \mathrm{e}-06$ & $6.09511 \mathrm{e}-06$ & $6.09511 \mathrm{e}-06$ \\
\hline 128 & $1.18934 \mathrm{e}-05$ & 0.24809 & 0.24809 & $9.74603 \mathrm{e}-06$ & $5.94671 \mathrm{e}-06$ & $5.94671 \mathrm{e}-06$ \\
\hline 256 & $1.15296 \mathrm{e}-05$ & 0.25532 & 0.25532 & $9.45829 \mathrm{e}-06$ & $5.76481 \mathrm{e}-06$ & $5.76481 \mathrm{e}-06$ \\
\hline
\end{tabular}

TABLE 3. As in Table 1, but with the noise variable set to $10^{-5} n^{-0.56}$.

If there is a unitary $Q$ and an upper-triangular matrix $T$ so that $V=Q T Q^{*}$, and if we define $D$ to be the diagonal unitary matrix with

$$
D_{j j}=\frac{1}{\left|T_{j j}\right|} T_{j j}
$$

then

$$
\left\|U-Q D Q^{*}\right\| \leq(\sqrt{2(n-1)}+2)\left\|U^{*} U-I\right\|
$$

Proof. We have

$$
\left\|V-Q D Q^{*}\right\| \leq(\sqrt{2(n-1)}+1)\left\|V^{*} V-I\right\|^{\frac{1}{2}} \leq(\sqrt{2(n-1)}+1)\left\|U^{*} U-I\right\|
$$

and

$$
\|U-V\| \leq\left\|U^{*} U-I\right\|
$$

It is worth keeping in mind that the spectral decomposition of the polar part of $U$ leads to the theoretical best unitary diagonalization, with $D$ diagonal, $Q$ unitary and

$$
\left\|U-Q^{*} D Q\right\|=\max \left\{|\lambda-1| \mid \lambda \in \sigma\left(\left(U^{*} U\right)^{\frac{1}{2}}\right)\right\}
$$

and the best general lower bound is

$$
\left\|U-Q^{*} D Q\right\| \geq \frac{1}{2}\left\|U^{*} U-I\right\| .
$$

Lemma 9. Suppose $U$ is in $\mathbf{M}_{n}(\mathbb{C})$ and $\left\|U^{*} U-I\right\| \leq \frac{3}{4}$. If

$$
V_{1}=\frac{1}{2}\left(U+\left(U^{-1}\right)^{*}\right)
$$

and

$$
V=\frac{1}{2}\left(V_{1}+\left(V_{1}^{-1}\right)^{*}\right)
$$




\begin{tabular}{|c|c|c|c|c|c|c|}
\hline & $\begin{array}{c}\text { deviation } \\
\text { from unitary }\end{array}$ & \multicolumn{5}{|c|}{ Backwards Error $\left\|e^{-i H}-U\right\|$} \\
\hline$n$ & $\left\|U^{*} U-I\right\|$ & Algorithm 1 & Algorithm 2 & Algorithm 3 & Algorithm 4 & Algorithm 5 \\
\hline \hline 8 & $3.98986 \mathrm{e}-01$ & 0.48072 & 0.48083 & $2.91776 \mathrm{e}-01$ & $1.87829 \mathrm{e}-01$ & $1.87829 \mathrm{e}-01$ \\
\hline 16 & $3.99794 \mathrm{e}-01$ & 0.76608 & 0.76629 & $3.00304 \mathrm{e}-01$ & $1.88836 \mathrm{e}-01$ & $1.88836 \mathrm{e}-01$ \\
\hline 32 & $4.01659 \mathrm{e}-01$ & 0.74454 & 0.74577 & $3.02891 \mathrm{e}-01$ & $1.86026 \mathrm{e}-01$ & $1.86026 \mathrm{e}-01$ \\
\hline 64 & $4.04556 \mathrm{e}-01$ & 0.81147 & 0.81217 & $2.99941 \mathrm{e}-01$ & $1.85677 \mathrm{e}-01$ & $1.85677 \mathrm{e}-01$ \\
\hline 128 & $3.92340 \mathrm{e}-01$ & 0.86934 & 0.87020 & $2.90461 \mathrm{e}-01$ & $1.79970 \mathrm{e}-01$ & $1.79970 \mathrm{e}-01$ \\
\hline 256 & $3.81114 \mathrm{e}-01$ & 1.23535 & 1.23575 & $2.84104 \mathrm{e}-01$ & $1.75207 \mathrm{e}-01$ & $1.75207 \mathrm{e}-01$ \\
\hline
\end{tabular}

TABLE 4. As in Table 1, but with the noise variable set to $0.3 n^{-0.56}$.

then

$$
\left\|V^{*} V-I\right\| \leq \frac{4}{25}\left\|U^{*} U-I\right\|^{4}
$$

and

$$
\|U-V\| \leq \frac{7}{10}\left\|U^{*} U-I\right\| .
$$

Proof. Tracking the spectrum of positive parts as before, we are looking at

$$
\frac{1}{2} \frac{\left(\frac{1}{2} \frac{\lambda^{2}+1}{\lambda}\right)^{2}+1}{\left(\frac{1}{2} \frac{\lambda^{2}+1}{\lambda}\right)}=\frac{\lambda^{4}+6 \lambda^{2}+1}{4 \lambda\left(\lambda^{2}+1\right)}
$$

and all that is needed is some algebra and calculus to verify that

$$
\frac{1}{2} \leq \lambda \leq \frac{\sqrt{7}}{2}
$$

implies

$$
\left|\left(\frac{\lambda^{4}+6 \lambda^{2}+1}{4 \lambda\left(\lambda^{2}+1\right)}\right)^{2}-1\right| \leq \frac{4}{25}\left|\lambda^{2}-1\right|^{4}
$$

and

$$
\left|\frac{\lambda^{4}+6 \lambda^{2}+1}{4 \lambda\left(\lambda^{2}+1\right)}-\lambda\right| \leq \frac{7}{10}\left|\lambda^{2}-1\right| .
$$

As before, we use this to get an estimate on the algorithm that uses two iterations of Newton's method followed by a Schur decomposition.

Theorem 10. Suppose $U$ is in $\mathbf{M}_{n}(\mathbb{C})$ with $\left\|U^{*} U-I\right\| \leq \frac{3}{4}$ and $n \geq 3$. Let

$$
V_{1}=\frac{1}{2}\left(U+\left(U^{-1}\right)^{*}\right)
$$


and

$$
V=\frac{1}{2}\left(V_{1}+\left(V_{1}^{-1}\right)^{*}\right)
$$

If there is a unitary $Q$ and an upper-triangular matrix $T$ so that $V=Q T Q^{*}$, and if we define $D$ to be the diagonal unitary matrix with

$$
D_{j j}=\frac{1}{\left|T_{j j}\right|} T_{j j}
$$

then

$$
\left\|U-Q D Q^{*}\right\| \leq \frac{7}{10} \sqrt{n}\left\|U^{*} U-I\right\|^{2}+\frac{7}{10}\left\|U^{*} U-I\right\| .
$$

Proof. We have

$$
\left\|V^{*} V-I\right\| \leq \frac{4}{25}\left\|U^{*} U-I\right\|^{4}
$$

and

$$
\begin{aligned}
\left\|V-Q D Q^{*}\right\| & \leq(\sqrt{2(n-1)}+1)\left\|V^{*} V-I\right\|^{\frac{1}{2}} \\
& \leq(\sqrt{2(n-1)}+1)\left(\frac{4}{25}\left\|U^{*} U-I\right\|^{4}\right)^{\frac{1}{2}} \\
& \leq \frac{7}{10} \sqrt{n}\left(\left\|U^{*} U-I\right\|^{4}\right)^{\frac{1}{2}}
\end{aligned}
$$

and

$$
\|U-V\| \leq \frac{7}{10}\left\|U^{*} U-I\right\|
$$

Rather extreme input data is need to highlight the advantage of Algorithm 5. We test algorithms 2,5 on unitaries, and approximate unitaries, that have multiple eigenvalues very near -1 . See Tables 1, 4 ,

Not much can be said regarding the error in the output $H$ of Algorithm 5 vs. the "true logarithm." The trouble is that the set of "exactly connect" answers jumps around in a most discontinious fashion. If $\alpha, \beta$ are in $(-\pi, \pi]$ with $\alpha \approx-\pi$ and $\beta \approx \pi$ then for any unitary $Q$, consider the unitary

$$
U=Q\left[\begin{array}{cc}
e^{i \alpha} & 0 \\
0 & e^{i \beta}
\end{array}\right] Q^{*}
$$

The only exactly correct output given $U$ on input is

$$
Q\left[\begin{array}{ll}
\alpha & 0 \\
0 & \beta
\end{array}\right] Q^{*}
$$

and this greatly depends on $Q$. 


\begin{tabular}{|c|c|c|c|c|}
\hline & $\begin{array}{c}\text { deviation } \\
\text { from unitary }\end{array}$ & \multicolumn{3}{|c|}{ Backwards Error $\left\|e^{-i H}-U\right\|$} \\
\hline$n$ & $\left\|U^{*} U-I\right\|$ & Algorithm 1A & Algorithm 2A & Algorithm 6 \\
\hline \hline 8 & $2.96904 \mathrm{e}-15$ & 1.17188 & 1.12520 & $3.27683 \mathrm{e}-15$ \\
\hline 16 & $3.27269 \mathrm{e}-15$ & 1.10402 & 1.11826 & $4.50363 \mathrm{e}-15$ \\
\hline 32 & $4.12604 \mathrm{e}-15$ & 1.13947 & 1.15103 & $6.68904 \mathrm{e}-15$ \\
\hline 64 & $8.33263 \mathrm{e}-15$ & 0.77344 & 0.77214 & $1.00208 \mathrm{e}-14$ \\
\hline 128 & $1.02179 \mathrm{e}-14$ & 1.46559 & 1.46102 & $1.52540 \mathrm{e}-14$ \\
\hline 256 & $2.46375 \mathrm{e}-14$ & 1.19984 & 1.19987 & $2.78177 \mathrm{e}-14$ \\
\hline
\end{tabular}

TABLE 5. We compare algorithms that produce matrices $H$ with $H^{*}=$ $H^{\sharp}=H$ exactly but with $e^{i H} \approx U$ with varying accuracy. These are run on unitary, $J$-skew-symmetric matrices with at least four eigenvalues near -1 . For each $n$ the results shown are averaged iver 30 test matrices. The noise variable was set to $10^{-15} n^{-0.56}$ in the code in the appendix.

\section{J-SKEW-SYMMETRIC UNITARIES}

When dealing with Hamiltonians for systems with certain time-reversal symmetry, we need an extra involution on matrices, the dual. Working in $N$-by- $N$ blocks, the dual operation on $n$-by- $n$ matrices with $n=2 N$ is defined as

$$
\left[\begin{array}{ll}
A & B \\
C & D
\end{array}\right]^{\sharp}=\left[\begin{array}{cc}
D^{\mathrm{T}} & -B^{\mathrm{T}} \\
-C^{\mathrm{T}} & A^{\mathrm{T}}
\end{array}\right] .
$$

It is easy to check this obeys the same axiom as the transpose. In particular it commutes with the adjoint. See [16], for example. The dual operation is not unique, but is fixed once we specify

$$
J=\left[\begin{array}{cc}
0 & I \\
-I & 0
\end{array}\right] .
$$

Then $X^{\sharp}=-J X^{\mathrm{T}} J$. A matrix $X$ is $J$-skew-symmetric when $X^{\sharp}=X$ and so if and only if $(X J)^{\mathrm{T}}=-X J$.

There is a $J$-skew-symmetric Schur decomposition result for $J$-skew-symmetric matrices, a.k.a. the skew-Hamiltonian Schur decomposition result from [2].

Theorem 11. Let $n=2 N$. If $X^{\sharp}=X$ in $\mathbf{M}_{n}(\mathbb{C})$ then there is a unitary $Q$ with $Q^{\sharp}=Q^{*}$ and a matrix $S$ with $X=Q S Q^{*}$ and so that

$$
S=\left[\begin{array}{cc}
T & B \\
0 & T^{\mathrm{T}}
\end{array}\right]
$$

where $T$ is upper triangular. Moreover there is an $O\left(n^{3}\right)$ algorithm to find $Q$ and $T$. 


\begin{tabular}{|c|c|c|c|c|}
\hline & $\begin{array}{c}\text { deviation } \\
\text { from unitary }\end{array}$ & \multicolumn{3}{|c|}{ Time } \\
\hline$n$ & $\left\|U^{*} U-I\right\|$ & Algorithm 1A & Algorithm 2A & Algorithm 6 \\
\hline \hline 8 & $2.96904 \mathrm{e}-15$ & $0.00019 \mathrm{~s}$ & $0.00223 \mathrm{~s}$ & $0.00250 \mathrm{~s}$ \\
\hline 16 & $3.27269 \mathrm{e}-15$ & $0.00034 \mathrm{~s}$ & $0.00503 \mathrm{~s}$ & $0.00616 \mathrm{~s}$ \\
\hline 32 & $4.12604 \mathrm{e}-15$ & $0.00124 \mathrm{~s}$ & $0.01752 \mathrm{~s}$ & $0.01492 \mathrm{~s}$ \\
\hline 64 & $8.33263 \mathrm{e}-15$ & $0.00773 \mathrm{~s}$ & $0.05169 \mathrm{~s}$ & $0.03858 \mathrm{~s}$ \\
\hline 128 & $1.02179 \mathrm{e}-14$ & $0.05879 \mathrm{~s}$ & $0.16380 \mathrm{~s}$ & $0.14007 \mathrm{~s}$ \\
\hline 256 & $2.46375 \mathrm{e}-14$ & $0.34309 \mathrm{~s}$ & $0.52146 \mathrm{~s}$ & $0.79723 \mathrm{~s}$ \\
\hline
\end{tabular}

TABLE 6. Timing data for the algorithms and data set from Table 5 .

Proof. This is the complex analog of [2, §2.1], discussed in detail in [10, §9.1]. One uses some variation on the Paige / Van Loan algorithm [2, §2.1], involving careful combinations of Givens rotations and partial Householder reflections to create a unitary $Q_{1}$ with $Q_{1}^{\sharp}=$ $Q_{1}^{*}$ and so that $X=Q_{1} S_{1} Q_{1}^{*}$ with

$$
S_{1}=\left[\begin{array}{cc}
T_{1} & B_{1} \\
0 & T_{1}^{\mathrm{T}}
\end{array}\right]
$$

An ordinary Schur decomposition $T_{1}=W T W^{*}$ now finishes the job, as

$$
Q=Q_{1}\left[\begin{array}{ll}
W & \\
& \bar{W}
\end{array}\right]
$$

has the needed symmetry, while

$$
S=\left[\begin{array}{ll}
W & \\
& \bar{W}
\end{array}\right]^{*} S_{1}\left[\begin{array}{ll}
W & \\
& \bar{W}
\end{array}\right]
$$

has the correct block structure.

For comparision purposes, we say we are using Algorithm 2A, etc, if we add a final step to the algorithms above that replaces $H$ by $\frac{1}{2} H^{\sharp}$.

Notice that $S$ becomes upper-triangular if we just reverse the order of half the basis elements.

Theorem 12. Let $n=2 N$. Suppose $U$ is a J-skew-symmetric matrix in $\mathbf{M}_{n}(\mathbb{C})$ and that unitary $Q$ and matrix $T$ form a J-skew-symmetric Schur decomposition for $U$. If we define $D$ to be the diagonal unitary matrix with

$$
D_{j j}=\frac{1}{\left|T_{j j}\right|} T_{j j}
$$

then $D$ is J-skew-symmetric, diagonal and

$$
\left\|U-Q D Q^{*}\right\| \leq(\sqrt{2(n-1)}+1)\left\|U^{*} U-I\right\|^{\frac{1}{2}} .
$$




\begin{tabular}{|c|c|c|c|c|}
\hline & $\begin{array}{c}\text { deviation } \\
\text { from unitary }\end{array}$ & \multicolumn{3}{|c|}{ Backwards Error $\left\|e^{-i H}-U\right\|$} \\
\hline$n$ & $\left\|U^{*} U-I\right\|$ & Algorithm 1A & Algorithm 2A & Algorithm 6 \\
\hline \hline 8 & $6.80865 \mathrm{e}-06$ & 0.50219 & 0.50219 & $3.40432 \mathrm{e}-06$ \\
\hline 16 & $7.91995 \mathrm{e}-06$ & 0.27931 & 0.27931 & $3.95997 \mathrm{e}-06$ \\
\hline 32 & $8.21901 \mathrm{e}-06$ & 0.32397 & 0.32397 & $4.10950 \mathrm{e}-06$ \\
\hline 64 & $8.30081 \mathrm{e}-06$ & 0.43814 & 0.43814 & $4.15041 \mathrm{e}-06$ \\
\hline 128 & $8.15713 \mathrm{e}-06$ & 0.30607 & 0.30607 & $4.07856 \mathrm{e}-06$ \\
\hline 256 & $8.02983 \mathrm{e}-06$ & 0.38732 & 0.38732 & $4.01491 \mathrm{e}-06$ \\
\hline
\end{tabular}

TABLE 7. As in Table 5, but with the noise variable set to $10^{-5} n^{-0.56}$.

If we approximate the polar part of $U$ by Newton's method, the symmetry $U^{\sharp}=U$ is preserved at each iteration, since

$$
\left(\frac{1}{2}\left(U+\left(U^{-1}\right)^{*}\right)\right)^{\sharp}=\frac{1}{2} U^{\sharp}+\left(\left(U^{-1}\right)^{*}\right)^{\sharp}=\frac{1}{2} U^{\sharp}+\left(\left(U^{-1}\right)^{\sharp}\right)^{*}=\frac{1}{2} U^{\sharp}+\left(\left(U^{\sharp}\right)^{-1}\right)^{*} .
$$

Theorem 13. Let $n=2 N$. Suppose $U^{\sharp}=U$ is in $\mathbf{M}_{n}(\mathbb{C})$ with $\left\|U^{*} U-I\right\| \leq \frac{3}{4}$ and let

$$
V_{1}=\frac{1}{2}\left(U+\left(U^{-1}\right)^{*}\right)
$$

and

$$
V=\frac{1}{2}\left(V_{1}+\left(V_{1}^{-1}\right)^{*}\right)
$$

If a unitary $Q$ and matrix $T$ form a J-skew-symmetric Schur decomposition for $V$, and if we define $D$ the diagonal unitary matrix with

$$
D_{j j}=\frac{1}{\left|T_{j j}\right|} T_{j j}
$$

then $D$ is $J$-skew-symmetric and

$$
\left\|U-Q D Q^{*}\right\| \leq \frac{7}{10} \sqrt{n}\left\|U^{*} U-I\right\|^{2}+\frac{7}{10}\left\|U^{*} U-I\right\| .
$$

Theorem 13 gives theoretical justification for that the following algorithm produces approximately correct output. This is very similar to the algorithm used in [17]. Since this is built out of known algorithms, we don't have anything to say it is $\mathcal{O}\left(n^{3}\right)$.

\section{Algorithm 6.}

(1) Set $V_{1}=\frac{1}{2}\left(U+\left(U^{-1}\right)^{*}\right)$.

(2) Set $V=\frac{1}{2}\left(V_{1}+\left(V_{1}^{-1}\right)^{*}\right)$.

(3) Compute a $J$-skew-symmetric Schur factorization, as in Theorem 13 , with $Q T Q^{*} \approx$ $V$.

(4) Create a unitary diagonal matrix $D$ via $D_{j j}=T_{j j} /\left|T_{j j}\right|$. 


\begin{tabular}{|c|c|c|c|c|}
\hline & $\begin{array}{c}\text { deviation } \\
\text { from unitary }\end{array}$ & \multicolumn{3}{|c|}{ Backwards Error $\left\|e^{-i H}-U\right\|$} \\
\hline$n$ & $\left\|U^{*} U-I\right\|$ & Algorithm 1A & Algorithm 2A & Algorithm 6 \\
\hline \hline 8 & $2.12731 \mathrm{e}-01$ & 0.45404 & 0.45282 & $1.05752 \mathrm{e}-01$ \\
\hline 16 & $2.43013 \mathrm{e}-01$ & 0.55252 & 0.55205 & $1.18737 \mathrm{e}-01$ \\
\hline 32 & $2.51907 \mathrm{e}-01$ & 0.83382 & 0.83331 & $1.21165 \mathrm{e}-01$ \\
\hline 64 & $2.61957 \mathrm{e}-01$ & 0.96758 & 0.96743 & $1.24065 \mathrm{e}-01$ \\
\hline 128 & $2.62333 \mathrm{e}-01$ & 0.94305 & 0.94325 & $1.23593 \mathrm{e}-01$ \\
\hline 256 & $2.56967 \mathrm{e}-01$ & 1.03853 & 1.03868 & $1.21172 \mathrm{e}-01$ \\
\hline
\end{tabular}

TABLE 8. As in Table 5, but with the noise variable set to $0.3 n^{-0.56}$.

\section{(5) Compute $Q \log (D) Q^{*}$.}

For data on timing and accuracy, see Tables 5, 8 . These tables were produced with the MATLAB code listed in the appendix. That code has almost no optimization. In particular, it does not take advantage of the symmetries $Q^{\sharp}=Q$ and $D^{\sharp}=D$ that hold at the top of the loop in the Paige - Van Loan algorithm.

\section{Logarithms in Physics}

In quantum mechanics, it is standard to exponential a skew-Hermitian operator to get a unitary, as this is how one moves from the Hamiltonian to the time evolution operator. When then is a periodic time-dependent Hamiltonian $H_{t}$ of period $T$, the definition of quasi-energy depends on the Floquet Hamiltonian $H_{F}$ defined via

$$
e^{-i H_{F}}=T e^{-i \int_{0}^{T} H_{t} d t} .
$$

It does not matter if the principal branch of logarithm is used to define $H_{F}$ but it is important in numerical studies that $H_{F}$ be computed to be Hermitian. The study of Floquet topological insulators [14] is an important special case of a system with a periodic time-dependent Hamiltonian. Some Floquet topological insulators can only be explained by keeping track of a form of time-reversal symmetry [13, equation 21]. In that case one has a self-dual Floquet Hamiltonian.

The logarithms of unitary matrices arise in another way in physics, in particular in the study of the more typical topological insulators where there is a time-independents Hamiltonian. For finite lattice models of non-interacting fermions, the dimension and symmetry class determine if distinct topological phases can occur. Physically these phases are ordinary insulators and topological insulators [8, 19].

Joint working with Hastings [10] established that the ordinary insulating phases can be characterized by the existence of localized vectors that form a basis of low-energy space (localized Wannier functions) where the basis preserves an appropriate symmetry. We 
explained in Section 4 of [10] how to translate this question into a question about almost normal, almost unitary, or almost commuting matrices.

The AII symmetry class is one where there is a certain flavor of time-reversal invariance. What this means mathematically is that one starts with $J$-skew-symmetric and Hermitian matrices for the Hamiltonian and the position observables. There is a promising method for computing the spin Chern numbers that involves self-dual logarithms. This was introduced in [17]. The formula in $K$-theory used there is now validated by the theorems in [15] and the results in [15].

\section{ACKNOWLEDGMents}

This work was partially supported by a grant from the Simons Foundation (208723 to Loring).

\section{REFERENCES}

[1] E. Anderson, Z. Bai, And C. Bischof, LAPACK Users' guide, vol. 9, Society for Industrial Mathematics, 1999.

[2] P. Benner, D. Kressner, and V. Mehrmann, Skew-Hamiltonian and Hamiltonian eigenvalue problems: Theory, algorithms and applications, in Proceedings of the Conference on Applied Mathematics and Scientific Computing, Springer, 2005, pp. 3-39.

[3] A. Bunse-Gerstner, R. Byers, and V. Mehrmann, A chart of numerical methods for structured eigenvalue problems, SIAM Journal on Matrix Analysis and Applications, 13 (1992), p. 419.

[4] S. Cheng, N. Higham, C. Kenney, And A. Laub, Return to the middle ages: A half-angle iteration for the logarithm of a unitary matrix, in Proceedings of the Fourteenth International Symposium of Mathematical Theory of Networks and Systems, Perpignan, France. CD ROM, 2000.

[5] K. Davidson, Essentially normal operators, A Glimpse at Hilbert Space Operators, (2010), pp. 209222.

[6] P. Davies and N. Higham, A Schur-Parlett algorithm for computing matrix functions.

[7] I. Fulga, F. Hassler, And A. Akhmerov, Scattering theory of topological insulators and superconductors, Arxiv preprint arXiv:1106.6351, (2011).

[8] I. Fulga, F. Hassler, A. Akhmerov, and C. Beenakker, Scattering formula for the topological quantum number of a disordered multimode wire, Physical Review B, 83 (2011), p. 155429.

[9] M. Hastings, Eigenvalue Distribution In The Self-Dual Non-Hermitian Ensemble, Journal of Statistical Physics, 103 (2001), pp. 903-913.

[10] M. B. Hastings And T. A. LoRing, Topological insulators and $C^{*}$-algebras: Theory and numerical practice, Ann. Physics, 326 (2011), pp. 1699-1759.

[11] P. Henrici, Bounds for iterates, inverses, spectral variation and fields of values of non-normal matrices, 4 (1962), pp. 24-40.

[12] N. Higham and P. Papadimitriou, A parallel algorithm for computing the polar decomposition, Parallel Computing, 20 (1994), pp. 1161-1173.

[13] Y. Katan And D. Podolsky, Modulated floquet topological insulators, Physical Review Letters, 110 (2013), p. 016802.

[14] N. Lindner, G. Refael, And V. Galitski, Floquet topological insulator in semiconductor quantum wells, Nature Physics, 7 (2011), pp. 490-495.

[15] T. A. Loring, Quantitative k-theory and spin chern numbers. arXiv:1302.0349,

[16] — Factorization of matrices of quaternions, Exposition. Math., 30 (2012), pp. 250-267.

[17] T. A. Loring And M. B. Hastings, Disordered topological insulators via $C^{*}$-algebras, Europhys. Lett. EPL, 92 (2010), p. 67004. 
[18] C. Moler and C. VAn LoAn, Nineteen dubious ways to compute the exponential of a matrix, SIAM review, 20 (1978), pp. 801-836.

[19] J. TEO AND C. Kane, Topological defects and gapless modes in insulators and superconductors, Physical Review B, 82 (2010), p. 115120.

\section{Appendix}

\subsection{Code for General Unitaries.}

function [unitary_error, time, accuracy] = testLogs (n, noise,NumbNewt)

time $=0$;

accuracy $=0$;

$\% \% \% \% \% \% \% \% \% \% \% \% \% \% \% \% \% \% \% \% \% \% \% \% \% \% \% \% \% \% \% \% \% \% \% \% \% \% \% \% \% \% \% \% \% \% \% \% \% \%$

$\%$ Create a test matrix, approximately unitary

$\%$ Not fully random as we want some spectrum near -1

$\% \% \% \% \% \% \% \% \% \% \% \% \% \% \% \% \% \% \% \% \% \% \% \% \% \% \% \% \% \% \% \% \% \% \% \% \% \% \% \% \% \% \% \% \% \% \% \% \% \% \% \% \% \% \% \% \%$

$\%$ exponentiate a random self-adjoint matrix to create a unitary

$K=0.25 *(\operatorname{rand}(n)+i * r a n d(n)-r a n d(n)-i * r a n d(n))$;

$\mathrm{K}=\mathrm{K}+\mathrm{K}^{\prime}$;

$\mathrm{K}=(4 * \mathrm{pi} / \operatorname{norm}(\mathrm{K})) * \mathrm{~K}$;

$\%$ exponentiate $i * K$ to get a unitary

$\mathrm{Q}=\operatorname{expm}(i * \mathrm{~K})$;

$\%$ create a "random" diagonal, to form the test unitary

$\mathrm{D}=\operatorname{diag}(\exp (2 * \mathrm{pi} * i *[0.5,0.5, \operatorname{rand}(1, \mathrm{n}-2)]))$;

$\mathrm{U}=\mathrm{Q} * \mathrm{D} * \mathrm{Q}^{\prime}$;

$\%$ add noise

$\mathrm{U}=\mathrm{U}+\mathrm{noi}$ se $*(\operatorname{rand}(\mathrm{n})+i * r a n d(\mathrm{n})-\operatorname{rand}(\mathrm{n})-i * r$ and $(\mathrm{n}))$;

unitary_error $=\operatorname{norm}\left(U^{\prime} * U-\operatorname{eye}(\operatorname{size}(U))\right)$;

$\% \% \% \% \% \% \% \% \% \% \% \% \% \% \% \% \% \% \% \% \% \% \% \% \% \% \% \% \% \% \% \% \% \% \% \% \% \% \% \% \% \% \% \% \% \% \% \% \% \% \% \%$

$\%$ Get a logarithm by diagonalizing $\mathrm{U}=\mathrm{W} * \mathrm{D} *$ inv $(\mathrm{A})$, then

$\%$ force it to be hermitian

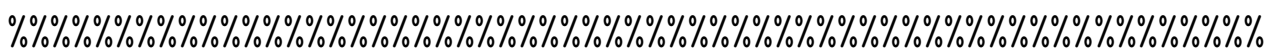

tstart = tic;

$[\mathrm{W}, \mathrm{D}]=\operatorname{eig}(\mathrm{U})$;

$X=\operatorname{diag}(\operatorname{imag}(\log (\operatorname{diag}(D))))$;

$\mathrm{H}=\mathrm{W} * \mathrm{X} * \operatorname{inv}(\mathrm{W})$;

$\mathrm{H}=(1 / 2) *\left(\mathrm{H}+\mathrm{H}^{\prime}\right)$;

time $(1)=\operatorname{toc}($ tstart $)$; 


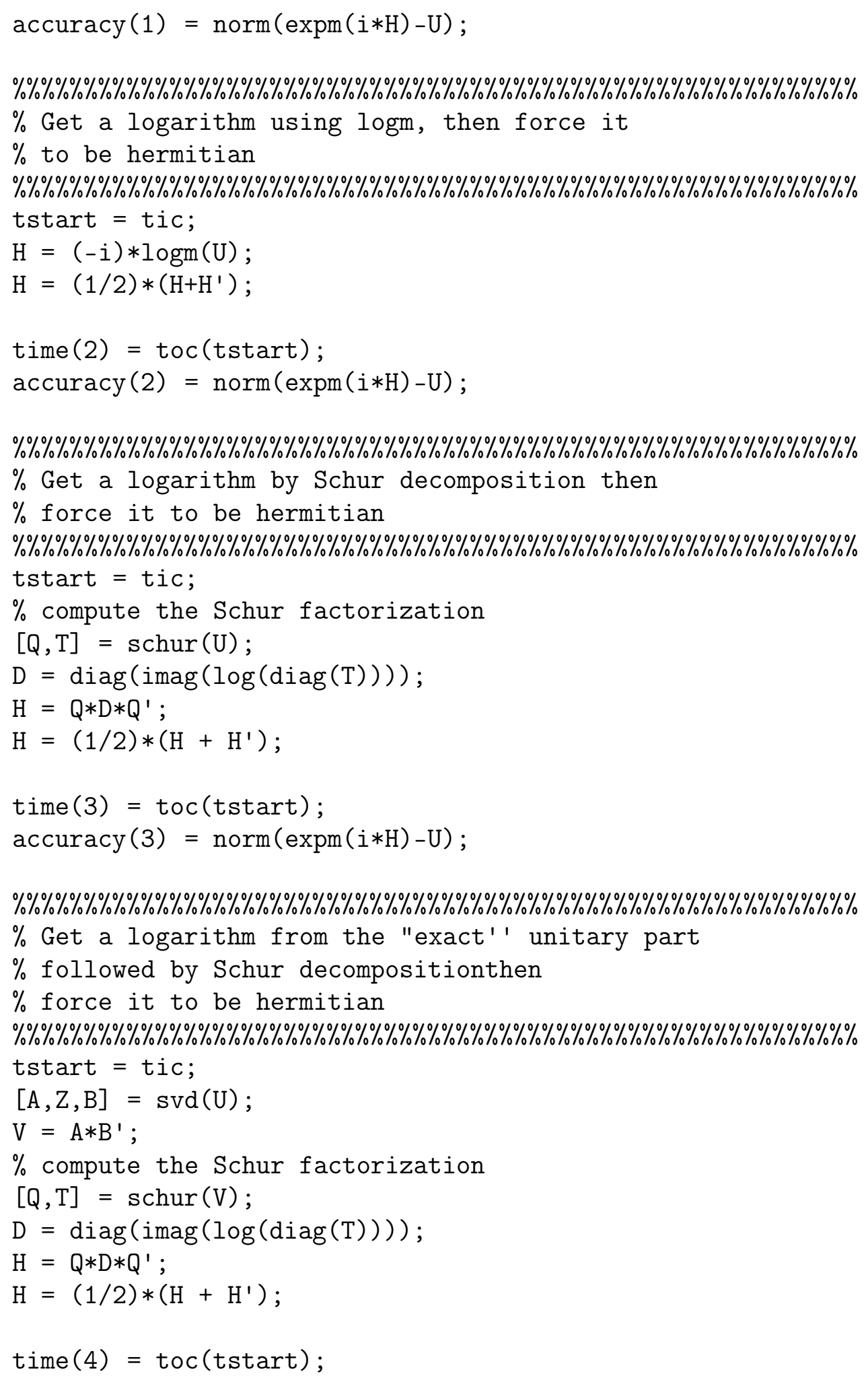




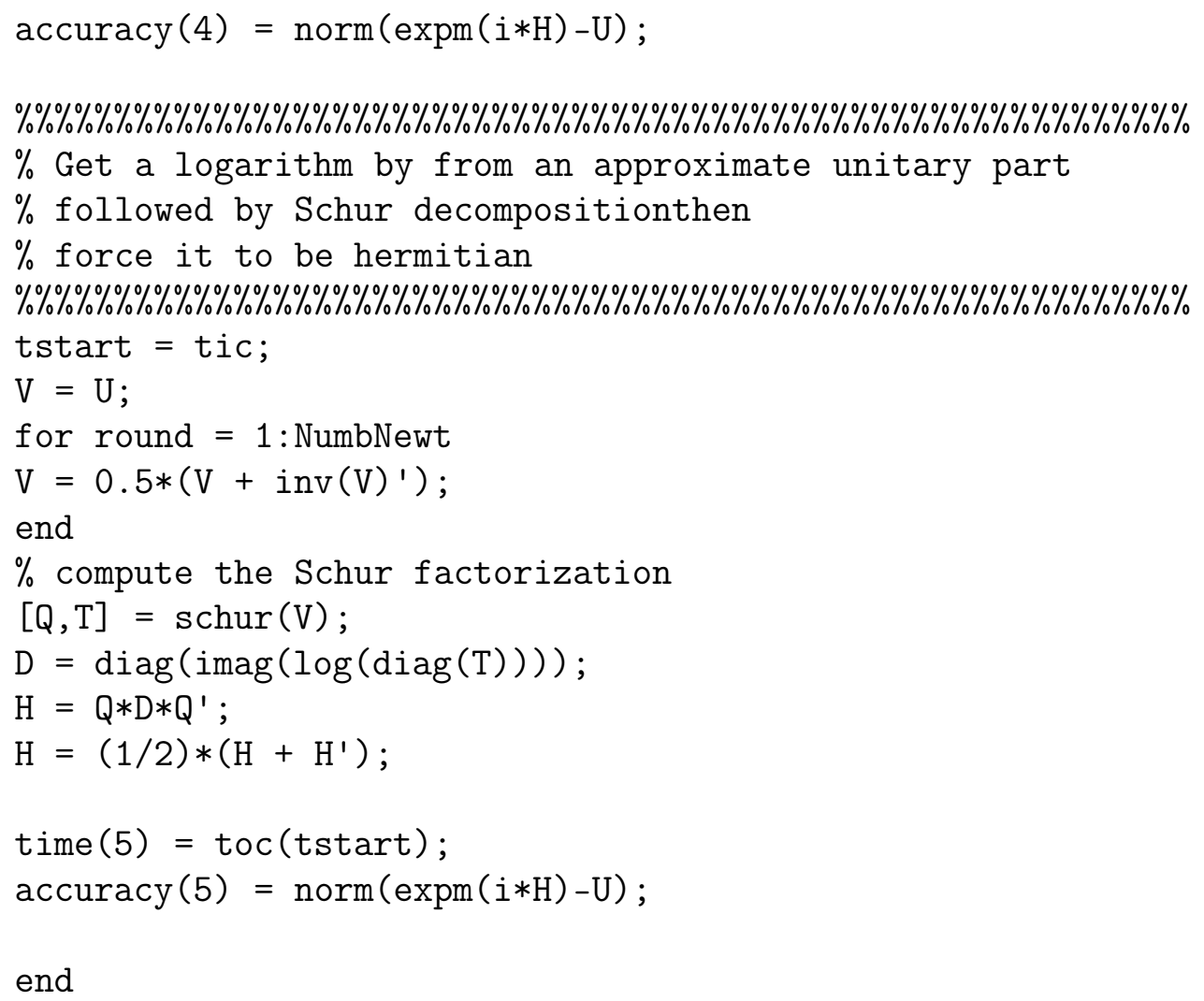


$\mathrm{K}=(1 / 2) *\left(\mathrm{~K}+\mathrm{K}^{\prime}\right)$;

$\mathrm{K}=(4 * \mathrm{pi} / \operatorname{norm}(\mathrm{K})) * \mathrm{~K}$;

$\%$ exponentiate $i * K$ to get a symplectic unitary

$Q=\operatorname{expm}(i * K)$;

$\%$ create a "random" diagonal, for form the test unitary

$\mathrm{D}=\exp (2 * \mathrm{pi} * \mathrm{i} *[0.5,0.5, \operatorname{rand}(1, \mathrm{~N}-2)])$;

$\mathrm{D}=\operatorname{diag}([\mathrm{D}, \mathrm{D}])$;

$\mathrm{U}=\mathrm{Q} * \mathrm{D} * \mathrm{Q}^{\prime}$;

$\%$ add noise, but keep self-dual

$\mathrm{U}=\mathrm{U}+$ noise $*(\operatorname{rand}(2 * \mathrm{~N})+\mathrm{i} * \mathrm{rand}(2 * \mathrm{~N})-\operatorname{rand}(2 * \mathrm{~N})-\mathrm{i} * \operatorname{rand}(2 * \mathrm{~N}))$;

$\mathrm{U}=(1 / 2) *(\mathrm{U}+\operatorname{dual}(\mathrm{U}))$;

unitary_error $=\operatorname{norm}\left(U^{\prime} * U-\operatorname{eye}(\operatorname{size}(U))\right)$;

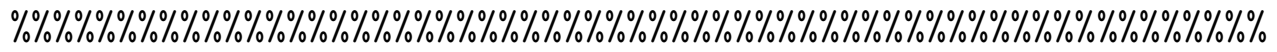

$\%$ Get a logarithm by diagonalizing $U=W * D * i n v(A)$, then

$\%$ force it to be hermitian and self dual

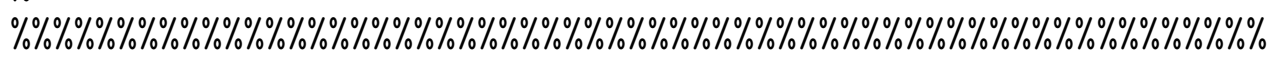

tstart = tic;

$[\mathrm{W}, \mathrm{D}]=\operatorname{eig}(\mathrm{U})$;

$\mathrm{X}=\operatorname{diag}(\operatorname{imag}(\log (\operatorname{diag}(D))))$;

$\mathrm{H}=\mathrm{W} * \mathrm{X} * \operatorname{inv}(\mathrm{W})$;

$\mathrm{H}=(1 / 2) *\left(\mathrm{H}+\mathrm{H}^{\prime}\right)$;

$\mathrm{H}=(1 / 2) *(\mathrm{H}+\operatorname{dual}(\mathrm{H}))$;

time $(1)=$ toc (tstart);

$\operatorname{accuracy}(1)=\operatorname{norm}(\operatorname{expm}(i * H)-U)$;

$\% \% \% \% \% \% \% \% \% \% \% \% \% \% \% \% \% \% \% \% \% \% \% \% \% \% \% \% \% \% \% \% \% \% \% \% \% \% \% \% \% \% \% \% \% \% \% \%$

$\%$ Get a logarithm using the default logm, then force it

$\%$ to be hermitian and self-dual

$\% \% \% \% \% \% \% \% \% \% \% \% \% \% \% \% \% \% \% \% \% \% \% \% \% \% \% \% \% \% \% \% \% \% \% \% \% \% \% \% \% \% \% \% \% \% \% \%$ tstart $=$ tic;

$\mathrm{H}=(-\mathrm{i}) * \operatorname{logm}(\mathrm{U})$;

$\mathrm{H}=(1 / 2) *\left(\mathrm{H}+\mathrm{H}^{\prime}\right)$;

$\mathrm{H}=(1 / 2) *(\mathrm{H}+\operatorname{dual}(\mathrm{H}))$;

time $(2)=$ toc (tstart);

$\operatorname{accuracy}(2)=\operatorname{norm}(\operatorname{expm}(i * H)-U)$;

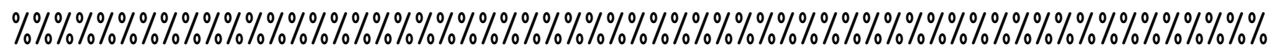


$\%$ Get a logarithm using Newton's method

$\%$ followed by a stuctured Schur decomposition, then force it

$\%$ to be hermitian and self-dual

$\% \% \% \% \% \% \% \% \% \% \% \% \% \% \% \% \% \% \% \% \% \% \% \% \% \% \% \% \% \% \% \% \% \% \% \% \% \% \% \% \% \% \% \% \% \% \% \% \% \% \% \% \% \% \% \% \%$

tstart $=$ tic;

\%Move toward a unitary with Newton's method

$\mathrm{V}=\mathrm{U}$;

for round $=1$ : NumbNewt

$\mathrm{V}=0.5 *\left(\mathrm{~V}+\operatorname{inv}(\mathrm{V})^{\prime}\right)^{\prime}$;

end

$\%$ compute the Schur factorization

$\%$ First the Paige - Van Loan algorithm

$[Q, D]=P V L(V)$;

$\mathrm{D}=\mathrm{D}(1: \mathrm{N}, 1: \mathrm{N})$;

$\%$ compute the little Schur factorization, finalize $Q$ and D.

$[\mathrm{Q} 1, \mathrm{~T} 1]=\mathrm{schur}(\mathrm{D})$;

$\mathrm{Q}(1: 2 * \mathrm{~N}, 1: \mathrm{N})=\mathrm{Q}(1: 2 * \mathrm{~N}, 1: \mathrm{N}) * \mathrm{Q} 1$;

$\mathrm{Q}(1: 2 * \mathrm{~N}, 1+\mathrm{N}: 2 * \mathrm{~N})=\mathrm{Q}(1: 2 * \mathrm{~N}, 1+\mathrm{N}: 2 * \mathrm{~N}) * \operatorname{conj}(\mathrm{Q} 1)$;

$\mathrm{D}=[\operatorname{diag}(\operatorname{imag}(\log (\operatorname{diag}(\mathrm{T} 1))))]$;

$\mathrm{D}=\left[\mathrm{D}, \operatorname{zeros}(\mathrm{N}) ; \operatorname{zeros}(\mathrm{N}), \mathrm{D} .{ }^{\prime}\right]$;

$H=Q * D * Q^{\prime}$;

$\mathrm{H}=(1 / 2) *\left(\mathrm{H}+\mathrm{H}^{\prime}\right)$;

$\mathrm{H}=(1 / 2) *(\mathrm{H}+\operatorname{dual}(\mathrm{H}))$;

time $(3)=$ toc (tstart);

$\operatorname{accuracy}(3)=\operatorname{norm}(\operatorname{expm}(i * H)-U)$;

end

$\%$ The Paige - Van Loan algorithm

function $[Q, D]=\operatorname{PVL}(V)$;

$\mathrm{n}=\operatorname{size}(\mathrm{V})$

$\mathrm{n}=\mathrm{n}(1) / 2$;

$Q=\operatorname{eye}(2 * n)$;

$\mathrm{D}=\mathrm{V} ; \%$ Expect to keep $\mathrm{V}=\mathrm{Q} * \mathrm{D} * \mathrm{Q}^{\prime}$

for $k=1:(n-1)$

\%Householder to fix most of the bottom half in column $k$ 
if $\mathrm{k}<(\mathrm{n}-1)$

$[\mathrm{v}$, beta $]=\operatorname{gallery}\left(\right.$ 'house' $\left.^{\prime} \mathrm{D}(\mathrm{k}+1+\mathrm{n}: 2 * \mathrm{n}, \mathrm{k})\right)$;

$\%$ on left of $\mathrm{D}$, top, then bottom

$\mathrm{D}(\mathrm{k}+1: \mathrm{n}, 1: 2 * \mathrm{n})=\mathrm{D}(\mathrm{k}+1: \mathrm{n}, 1: 2 * \mathrm{n}) \ldots$

- $(\operatorname{conj}($ beta $) * \operatorname{conj}(v)) *(\operatorname{conj}(v) ' * D(k+1: n, 1: 2 * n))$;

$\mathrm{D}(\mathrm{k}+1+\mathrm{n}: 2 * \mathrm{n}, 1: 2 * \mathrm{n})=\mathrm{D}(\mathrm{k}+1+\mathrm{n}: 2 * \mathrm{n}, 1: 2 * \mathrm{n}) \ldots$

- (beta*v)*( $\left.\mathrm{v}^{\prime} * \mathrm{D}(\mathrm{k}+1+\mathrm{n}: 2 * \mathrm{n}, 1: 2 * \mathrm{n})\right)$;

$\%$ on right of $\mathrm{D}$, top, then bottom

$\mathrm{D}(1: 2 * \mathrm{n}, \mathrm{k}+1: \mathrm{n})=\mathrm{D}(1: 2 * \mathrm{n}, \mathrm{k}+1: \mathrm{n}) \ldots$

- (beta* $(D(1: 2 * n, k+1: n) * \operatorname{conj}(v))) * \operatorname{conj}(v)^{\prime}$;

$\mathrm{D}(1: 2 * \mathrm{n}, \mathrm{k}+1+\mathrm{n}: 2 * \mathrm{n})=\mathrm{D}(1: 2 * \mathrm{n}, \mathrm{k}+1+\mathrm{n}: 2 * \mathrm{n}) \ldots$

- $(\operatorname{conj}($ beta $) *(D(1: 2 * n, k+1+n: 2 * n) * v)) * v^{\prime}$;

$\%$ on right of $Q$

$\mathrm{Q}(1: 2 * \mathrm{n}, \mathrm{k}+1: \mathrm{n})=\mathrm{Q}(1: 2 * \mathrm{n}, \mathrm{k}+1: \mathrm{n}) \ldots$

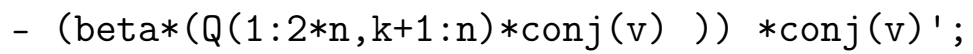

$\mathrm{Q}(1: 2 * \mathrm{n}, \mathrm{k}+1+\mathrm{n}: 2 * \mathrm{n})=\mathrm{Q}(1: 2 * \mathrm{n}, \mathrm{k}+1+\mathrm{n}: 2 * \mathrm{n}) \ldots$

- $(\operatorname{conj}($ beta $) *(\mathrm{Q}(1: 2 * \mathrm{n}, \mathrm{k}+1+\mathrm{n}: 2 * \mathrm{n}) * \mathrm{v})) * \mathrm{v}^{\prime}$;

end

$\% \mathrm{~A}$ symplectic Givens rotation to clear out $\mathrm{D}(\mathrm{k}+1+\mathrm{n}, \mathrm{k})$

$[\mathrm{G}, \mathrm{y}]=\operatorname{planerot}([\mathrm{D}(\mathrm{k}+1, \mathrm{k}) ; \mathrm{D}(\mathrm{k}+1+\mathrm{n}, \mathrm{k})])$;

$\%$ on left of $\mathrm{D}$

top_row $=\mathrm{D}(\mathrm{k}+1,1: 2 * \mathrm{n})$;

bottom_row $=\mathrm{D}(\mathrm{k}+1+\mathrm{n}, 1: 2 * \mathrm{n})$;

$\mathrm{D}(\mathrm{k}+1,1: 2 * \mathrm{n})=\mathrm{G}(1,1) *$ top_row $+\mathrm{G}(1,2) *$ bottom_row

$\mathrm{D}(\mathrm{k}+1+\mathrm{n}, 1: 2 * \mathrm{n})=\mathrm{G}(2,1) *$ top_row $+\mathrm{G}(2,2) *$ bottom_row

$\%$ on right of $\mathrm{D}$

left_col $=\mathrm{D}(1: 2 * \mathrm{n}, \mathrm{k}+1)$;

right_col $=\mathrm{D}(1: 2 * \mathrm{n}, \mathrm{k}+1+\mathrm{n})$;

$\mathrm{D}(1: 2 * \mathrm{n}, \mathrm{k}+1)=\operatorname{conj}(\mathrm{G}(1,1)) *$ left_col $_{\text {econj }}(\mathrm{G}(1,2)) *$ right_col ;

$\mathrm{D}(1: 2 * \mathrm{n}, \mathrm{k}+1+\mathrm{n})=\operatorname{conj}(\mathrm{G}(2,1)) * \operatorname{left}_{-} \operatorname{col}+\operatorname{conj}(\mathrm{G}(2,2)) * r i g h t_{-} \mathrm{col}$;

$\%$ on right of $Q$

left_col $=\mathrm{Q}(1: 2 * \mathrm{n}, \mathrm{k}+1)$;

right_col $=\mathrm{Q}(1: 2 * \mathrm{n}, \mathrm{k}+1+\mathrm{n})$;

$Q(1: 2 * n, k+1)=\operatorname{conj}(G(1,1)) *$ left_col+conj $(G(1,2)) * r i g h t_{-} \operatorname{col}$;

$\mathrm{Q}(1: 2 * \mathrm{n}, \mathrm{k}+1+\mathrm{n})=\operatorname{conj}(\mathrm{G}(2,1)) * \operatorname{left}_{\text {c }} \operatorname{col}+\operatorname{conj}(\mathrm{G}(2,2)) * \mathrm{right} \_\mathrm{col}$;

$\%$ Householder to fix top half in column $\mathrm{k}$

if $\mathrm{k}<(\mathrm{n}-1)$

$[\mathrm{v}$, beta $]=$ gallery $\left(\right.$ 'house $\left.^{\prime}, \mathrm{D}(\mathrm{k}+1: \mathrm{n}, \mathrm{k})\right)$;

$\%$ on left of $D$, top, then bottom

$\mathrm{D}(\mathrm{k}+1: \mathrm{n}, 1: 2 * \mathrm{n})=\mathrm{D}(\mathrm{k}+1: \mathrm{n}, 1: 2 * \mathrm{n}) \ldots$ 


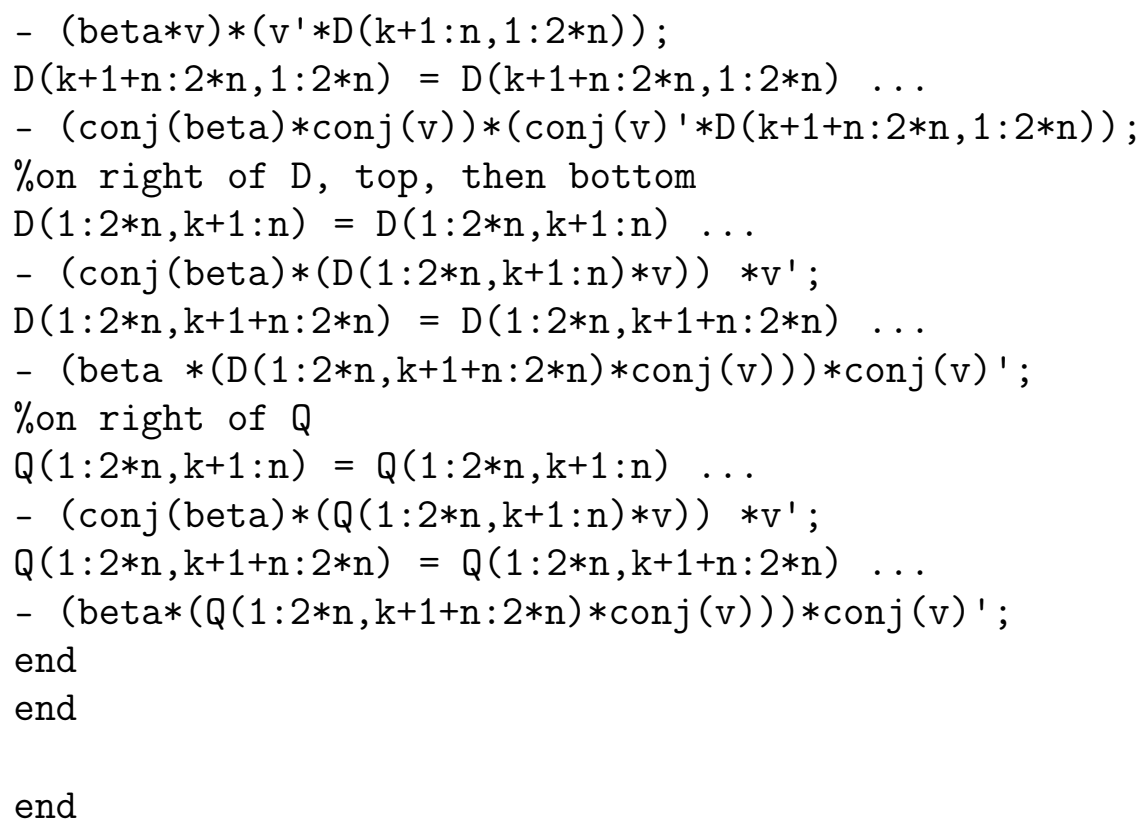

Department of Mathematics and Statistics, University of New Mexico, Albuquerque, NM 87131, USA. 